\title{
ON A (PSEUDO)HOLOGRAPHIC NATURE OF THE SYK-LIKE MODELS
}

\author{
D.V. Khveshchenko \\ Department of Physics and Astronomy, University of North Carolina, Chapel Hill, NC 27599, U.S.A \\ Email: khvesh@physics.unc.edu
}

Received 13 May 2019; accepted 21 June 2019

\begin{abstract}
Some generalizations of the Sachdev-Ye-Kitaev (SYK) model and different patterns of their reparametrization symmetry breaking are discussed. The analysis of such (pseudo)holographic systems relates their generalized onedimensional Schwarzian dynamics to (quasi) two-dimensional Liouvillian quantum mechanics. As compared to the original SYK case, the latter might be dissipative or have discrete states in its spectrum, either of which properties alters thermodynamics and correlations while preserving the underlying $S L(2, R)$ symmetry.
\end{abstract}

Keywords: Sachdev-Ye-Kitaev (SYK) model, holographic systems, Schwarzian dynamics

The 1+0-dimensional (quantum mechanical) SYK model of a large number $N$ of the Dirac (complex) [1-3] or Majorana (real) [4-7] fermions with all-to-all random couplings and its various generalizations (including non-random ones) [8-12] have attracted much attention lately. Those models are often cited as much-needed controllable examples of holographic correspondence which are expected to be dual to some 1+1-dimensional gravity (plus, possibly, extra fields).

Apart from remaining one of the central driving forces of modern string and high energy theory, the highly non-trivial and intriguing idea of (possibly, generalized beyond the original case of $A d S_{5} /$ $\mathrm{CFT}_{4}$ ) holography has already permeated other fields, including condensed matter physics. Over the past decade, a staggering number of holographic calculations alleged to be relevant to the realistic 'strange metals' and other complex ('non-Fermiliquid') systems has emerged [13-15].

However, despite the invariably upbeat claims of some of its enthusiastic practitioners, the use of the holographic technique outside of the original (subjected to a number of stringent constraints) string-theoretical context still remains to be justified and the true status of the cornucopia of look-alike and customarily verbose exercises in (generalized) classical relativity known under the acronym AdS/ $C M T$ is yet to be ascertained.

In a sharp contrast with such 'per analogiam' (a.k.a. 'bottom up') approach, the SYK model seemed to offer an example of fully controllable holographic correspondence between two theories of different dimensionalities that might both be amenable to the (asymptotically) exact analytical treatments. Also, despite its solubility, the SYK model was shown to be maximally chaotic, akin to black holes [4-7, 16-24], thus potentially providing insight into the inner workings of the generalized holographic conjecture and contributing towards its ultimate verification.

It is known, though, that the (nominally $2 d$ ) Jackiw-Teitelboim (JT) dilaton gravity that was conjectured as the SYK's bulk dual [4-7, 16-24] lacks any dynamical bulk degrees of freedom and, in fact, reduces to the theory of a fluctuating $1 d$ boundary. Indeed, the only available solution with constant (negative) curvature $R=-2$ implies the rigid $\mathrm{AdS}_{2}$ metric. Correspondingly, the JT spectrum turns out to be quite different from the 'dimension gap-free' SYK's one and in order to reconcile between the two an infinite tower of additional massive bulk scalar fields needs to be introduced [16-24]. 
Thus, while important and insightful, the SYK-JT relationship may not quite rise to the same level as a would-be 'bona fide' holographic correspondence between two theories operating irreducibly (yet, in both cases, locally) in different dimensions [1315. Instead, the SYK-JT correspondence where the gravitational background metric appears to be essentially non-dynamical could be viewed as a case of 'holography light' - a less ambitious scenario [25, 26] that so far has been largely ignored.

As a matter of fact, nearly all of the previous 'bottom up' holographic calculations [13-15] have been performed for certain fixed (usually, wellstudied) background metrics while neglecting any potential $O(1 / N)$ (here $N$ stands for the rank of an internal symmetry group, if any) corrections - either matter's backreaction upon gravity or quantum fluctuations of the latter.

Notably, though, the thus-obtained results would then be used to seek (and often claimed to have found) a good (including quantitative) agreement with some pre-selected sets of data on the physical systems with $N \sim 1$. Conceivably, if indeed present, such a fortuitous agreement would seem to indicate that no metric fluctuations should have been allowed in the first place.

Apart from the continuing exploration of such salient features of the original SYK model as its maximally chaotic behaviour [4-7, 16-24], some of the recent work has been challenging the various popular simplifying assumptions, such as replica symmetry of the SYK solutions [27-30]. Also, a technically related issue of the possibility of spatially dispersive solutions has been raised [31, 32] in the context of the various multi-dimensional 'SYK-lattice' generalizations which would be routinely assumed to remain spatially ultralocal due to the (presumed to always remain intact) local $Z_{2}$ symmetry [33-36].

The work on the SYK generalizations targets those behaviours that could survive departures from the original model, thus manifesting potentially generic, rather than unique to the SYK model, properties. In the present note, this quest is further pursued towards the different patterns of such central to the SYK issue as reparametrization symmetry breaking and the associated sequence of SYK-like models.

By analogy with the original SYK treatment [1-7, 16-24] a convenient starting point can be chosen in the form of a $1+0$-dimensional path integral over a pair of bi-local field variables $G\left(\tau_{1}, \tau_{2}\right)$ and $\Sigma\left(\tau_{1}, \tau_{2}\right)$

$$
\begin{aligned}
Z=\int & \operatorname{DGD} \Sigma\left(\operatorname{Det}\left[F\left[\partial_{\tau}\right]+\Sigma\right]\right)^{N} \\
& \times \exp \left(N \int_{\tau_{1}, \tau_{2}} G \Sigma-A[G]\right),
\end{aligned}
$$

where $F$ and $A$ are some functionals of their (operator-valued) arguments and the thermodynamics time varies within the interval $0 \leq \tau \leq 1 / \beta$. Depending on the nature of the underlying fundamental fields - e.g. complex vs real fermions - the variables $G$ and $\Sigma$ may have certain symmetrical properties as well. In what follows they will be treated as real-valued, consistent with the Majorana case. A generic functional

$$
A=N \sum_{k}^{\infty} \int_{\tau_{1}, \ldots \tau_{k}} J_{k}^{2}\left(\tau_{1}, \ldots \tau_{k}\right) G q\left(\tau_{1}, \tau_{2}\right) \ldots G^{q}\left(\tau_{k-1}, \tau_{k}\right)
$$

reproduces the '2q-interacting' SYK model for $J_{k}^{2} \sim \delta k_{2}$ (in contrast to its original formulation [1-7], here $q$ is an arbitrary - not necessarily even - integer). In fact, the action (2) is not unique to the original SYK model but can also describe its non-random cousins [8-12].

From the general standpoint, the applicability of Eq. (2) requires asymptotic dominance of the 'chain-melonic' diagrams. For a prospective microscopic system such behaviour (or a lack thereof) could, in principle, be established by extending the analysis [37, 38] of the SYK diagrammatics which is dominated by the ordinary 'melonic' $(k=2)$ graphs. The standard renormalization argument suggests, however, that in the absence of a special fine tuning of the parameters $J_{k}$ the infrared (IR) dynamics of the theory $(1,2)$ is still likely to be governed by the lowest dimension term (i.e. $k=2$ ).

Varying Eq. (1) with respect to $G$ and $\Sigma$ one obtains the mean-field equations

$$
\begin{aligned}
& \int_{\tau}\left(F\left(\partial_{\tau}\right) \delta\left(\tau_{1}, \tau\right)+\Sigma\left(\tau_{1}, \tau\right)\right) G\left(\tau, \tau_{2}\right)=\delta\left(\tau_{1}-\tau_{2}\right), \\
& \Sigma\left(\tau_{1}, \tau_{2}\right)=\frac{1}{N} \frac{\delta A}{\delta G\left(\tau_{1}, \tau_{2}\right)} .
\end{aligned}
$$

Neglecting the time derivatives completely, one finds that the saddle points of (1) are given by the solutions of the integral equation 


$$
\int_{\tau} G\left(\tau_{1}, \tau\right) \frac{\delta A}{\delta G\left(\tau_{1}, \tau_{2}\right)}=\delta\left(\tau_{1}-\tau_{2}\right),
$$

which appears to be manifestly invariant under the infinite group $\operatorname{Diff}\left(S^{1}\right)$ of reparametrizations of the thermal circle $\tau \rightarrow f(\tau)$ with the periodicity condition $f(\tau+\beta)=f(\tau)+\beta$, provided that $G$ and $\Sigma$ transform as

$$
\begin{aligned}
& G\left(\tau_{1}, \tau_{2}\right) \rightarrow G_{f}=\left[f^{\prime}\left(\tau_{1}\right) f^{\prime}\left(\tau_{2}\right)\right]^{\Delta} G\left(f\left(\tau_{1}\right), f\left(\tau_{2}\right)\right), \\
& \Sigma\left(\tau_{1}, \tau_{2}\right) \rightarrow \Sigma_{f}=\left[f^{\prime}\left(\tau_{1}\right) f^{\prime}\left(\tau_{2}\right)\right]^{1-\Delta} \Sigma\left(f\left(\tau_{1}\right), f\left(\tau_{2}\right)\right),
\end{aligned}
$$

where $\Delta=1 / 2 q$.

In the SYK case Eq. (4) permits a translationallyinvariant 'conformal' solution (here $\delta \tau_{12}=\tau_{1}-\tau_{2}$ ) 1-7]

$$
G_{0}\left(\tau_{1}, \tau_{2}\right)=\left(\frac{\pi}{\beta \sin \left(\pi \delta \tau_{12} / \beta\right)}\right)^{2 \Delta}
$$

which spontaneously breaks the full reparametrization symmetry down to its three-dimensional subgroup $S L(2, R)$ implemented through the Möbius transformations

$$
\tan \frac{\pi f(\tau)}{\beta} \rightarrow \frac{a \tan \frac{\pi f(\tau)}{\beta}+b}{c \tan \frac{\pi f(\tau)}{\beta}+d}
$$

with $a d-b c=1$, under which the function (6) (hence, the entire action given by Eqs. $(1,2)$ ) remains invariant.

The rest of the group $\operatorname{Diff}\left(S^{1}\right) / S L(2, R)$ then extends Eq. (6) onto the coadjoint Virasoro orbit where the dynamics of the field variable $f(\tau)$ is governed by the (sub-dominant) non-reparametrization invariant action $(1,2)$.

In the original SYK model $\left(F(x)=x, J_{k}=\delta_{k 2}\right)$ the leading (albeit IR-irrelevant in the RG sense) term of order $O(N / \beta J)$ stems from the first time derivative in the (Pfaffian) determinant

$$
A_{0}=\operatorname{Tr} \ln \left(1-\partial_{\tau} G_{f}\right)=-M \int_{\tau} \operatorname{Sch}\left\{\tan \frac{\pi f}{\beta}, \tau\right\}
$$

and is controlled by the characteristic time scale $M=\alpha_{s} N / J$ proportional to the numerically computed (q-dependent) prefactor $\alpha_{s}$ [4-7, 16-24]. The integrand readily identifies with the manifestly geometrical and $S L(2, R)$-invariant Schwarzian derivative

$$
\operatorname{Sch}\{F, \tau\}=\frac{F^{\prime \prime \prime}}{F^{\prime}}-\frac{3}{2}\left(\frac{F^{\prime \prime}}{F^{\prime}}\right)^{2}
$$

which satisfies the differential 'chain rule', $\operatorname{Sch}\{F(f), \tau\}=\operatorname{Sch}\{F, f\} f^{\prime 2}+\operatorname{Sch}\{f, \tau\}$, when applied to a composite function, such as $F(f(\tau))=\tan \pi f(\tau) / \beta$ (alternatively, the thermal circle can also be parametrized in terms of the function $\mathrm{e}^{\mathrm{i} \pi f / \beta}$ [4-7].

The emergence of the Schwarzian is to be expected as it controls the short-time expansion of the transformed solution (6) (here $\tau=\left(\tau_{1}+\tau_{2}\right) / 2$ )

$$
\begin{aligned}
& \delta G=G_{f}\left(\tau_{1}, \tau_{2}\right)-G_{0}\left(\tau_{1}, \tau_{2}\right) \approx \\
& \approx \frac{\Delta}{6}\left(\delta \tau_{12}\right)^{2} \operatorname{Sch}\{f, \tau\} G_{0}\left(\tau_{1}, \tau_{2}\right)+\ldots
\end{aligned}
$$

The standard mean-field ('large- $N$ ') SYK scenario [1-7] sets in for $1 / J \ll \beta \ll M$ where the fluctations $\delta G$ about the solution (6) are negligible. In contrast, for $M \lesssim \beta$ these fluctuations grow strong, thereby modifying the mean-field behaviour [39, 40].

Notably, the next, $O\left(N /(\beta J)^{2}\right)$, order correction to Eq. (8) is no longer local [4-7],

$$
\delta A \sim \frac{N}{J^{2}} \int_{\tau_{1} \tau_{2}} \frac{\left(f_{1}^{\prime} f_{2}^{\prime}\right)^{2}}{\left(\delta \tau_{12}\right)^{4}} \ln \left(\frac{J^{2}\left(\delta \tau_{12}\right)^{2}}{f_{1}^{\prime} f_{2}^{\prime}}\right),
$$

so the holographic postulate of locality holds only in the leading approximation.

Moreover, in addition to the 'gradient' terms, such as Eq. (8), the reparametrization symmetry can also be broken by choosing more generic timedependent couplings $J_{k}$ in Eq. (2). Furthermore, for certain couplings the $S L(2, R)$-invariant Eq. (6) still remains a solution as, e.g. in the case of

$$
J_{k}^{2}(\delta \tau)=\delta_{k, 2} \frac{J^{2-2 \gamma}}{(\delta \tau)^{2 \gamma}},
$$

although $\gamma>0$ alters the anomalous field dimension to

$$
\Delta=\frac{1-\gamma}{2 q} .
$$

The low-energy soft-mode action then gets modified by a non-local term which for $\gamma \ll 1$ can be approximated as a quadratic one (here $\Gamma=2 q \gamma N J \Delta / 3$ ) 


$$
\begin{aligned}
& \delta A= \\
& \frac{\Gamma}{J} \int_{\tau_{\tau} \tau_{2}}\left(\delta \tau_{12}\right)^{2} \ln \left(J \delta \tau_{12}\right) G_{f}^{2 q}\left(\tau_{1}, \tau_{2}\right) S \operatorname{ch}\left\{\tan \frac{\pi f}{\beta}, \tau\right\} \approx \\
& \frac{\Gamma}{J} \int_{\tau_{1} \tau_{2}} \frac{\left(f_{1}^{\prime}-1\right)\left(f_{2}^{\prime}-1\right)}{\left(\delta \tau_{12}\right)^{2}} .
\end{aligned}
$$

and is reminiscent of the ohmic dissipation in the Caldeira-Leggett model.

Among other things, the underlying $S L(2, R)$ algebraic structure suggests a systematic way of extending the conjectured (pseudo)holographic SYK-JT connection from the pure Schwarzian (8) to a broader class of the $1 \mathrm{~d}$ boundary theories the action of which may include Eqs. (11, 14), etc. They can be conveniently formulated in terms of the Hamiltonian dynamics on the $4 \mathrm{~d}$ phase space spanned by two pairs of canonically conjugated variables, $\left(f, \pi_{f}\right)$ and $\left(\phi, \pi_{\phi}\right)$, the former one being the aforementioned boundary 'conformal' time [41-45].

A pertinent Hamiltonian then conforms to the $S L(2, R)$-invariant quadratic Casimir operator

$$
H=\frac{1}{2} L_{0}^{2}-\frac{1}{4}\left(L_{1} L_{-1}+L_{-1} L_{1}\right)
$$

where the $S L(2, R)$ generators $L_{0, \pm 1}$ obey the Poisson brackets algebra

$$
\left\{L_{0}, L_{ \pm 1}\right\}= \pm L_{ \pm 1},\left\{L_{-1}, L_{1}\right\}=2 L_{0} .
$$

The various realizations of this algebra allow one to construct a host of dual boundary systems.

For instance, the general Hamiltonian (15) constructed with the use of the ansatz

$$
\begin{aligned}
& L_{-1}=\pi_{f}, \quad L_{0}=f \pi_{f}+\pi_{\phi}, \\
& L_{1}=f^{2} \pi_{f}+2 f \pi_{\phi}+A(\phi)-B(\phi) \pi_{f}-\frac{C(\phi)}{\pi_{f}}
\end{aligned}
$$

describes a charged non-relativistic particle of unit mass confined to a $2 d$ surface with some (diagonal) metric $g_{i j}=\operatorname{diag}\left[g_{\phi \phi}, g_{f f}\right]$ and subjected to the static vector $\left(A_{\phi}, A_{f}\right)$ and scalar $\Phi$ potentials,

$$
H=\frac{1}{4} g^{\phi \phi} \pi_{\phi}^{2}+\frac{1}{2} g^{f f}\left(\pi_{f}-A_{f}\right)^{2}+\Phi,
$$

where the background fields are given by the expressions

$$
\begin{aligned}
& g^{i j}=\operatorname{diag}[1, B(\phi)], \\
& A_{i}=\left(0, \frac{A(\phi)}{B(\phi)}\right), \quad \Phi=C(\phi)-\frac{A(\phi)^{2}}{4 B(\phi)} .
\end{aligned}
$$

The coordinate $f$ appears to be cyclic, so that the conjugate momentum $\pi_{f}$ is conserved. In fact, even for generic vector $A_{\mathrm{i}}(\phi)$ and scalar $\Phi(\phi)$ potentials the dynamics described by the Hamiltonian (18) remains effectively one-dimensional (this observation would have been far less obvious, though, had the vector potential been taken in a gauge other than the Landau one).

Moreover, for $A(\phi)=2 a \mathrm{e}^{\phi}, B(\phi)=b \mathrm{e}^{2 \phi}$, and $C(\phi)=c$ the operators (17) obey the algebra (16), thus guaranteeing the $S L(2, R)$-invariance of Eq. (15), which now takes the form

$$
H=\frac{1}{2} \pi_{\phi}^{2}+\frac{b}{2} \pi_{f}^{2} \mathrm{e}^{2 \phi}-a \mathrm{e}^{\phi} \pi_{f}+\frac{1}{2} c,
$$

while the metric $\mathrm{d} s^{2}=\mathrm{d} \phi^{2}+\mathrm{e}^{-2 \phi} \mathrm{d} f^{2}$ becomes that of the hyperbolic plane $H^{2}$.

Such connection between the SYK problem and a particle on $\mathrm{H}^{2}$ in magnetic field has been pointed out and exploited before [4-7, 41-45]. As the above suggests, it can be extended towards a broader class of (ostensibly) $2 d$ Hamiltonians - albeit, at the expense of adding new (admittedly, somewhat unphysical) terms proportional to the powers of momentum $\pi_{f}^{n}$ with $n>2$ and/or $n<0$.

The standard SYK scenario corresponds to choosing $a \pi_{f}=-\mu, b=c=0$, which reduces (20) to the Hamiltonian of the $1 d$ Liouville quantum mechanics

$$
H=\frac{1}{2} \pi_{\phi}^{2}+\mu \mathrm{e}^{\phi},
$$

the relation of which to the Schwarzian action (8) has been discussed extensively [39-45]. Indeed, upon the substitution $f^{\prime}=\mathrm{e}^{\phi}$ the zero-temperature action (8) amounts to the Gaussian kinetic energy of the (unbounded) variable $\phi(\tau)$, since $\operatorname{Sch}\{f, \tau\}=\left(\partial_{\tau} \phi\right)^{2}$.

Moreover, this change of variables can be formally implemented as a constraint enforced by the momentum $\pi$ playing the role of the Lagrange multiplier [39-45]. Introducing the second momentum $\pi_{\phi}$ in the Legendre transformation of the Schwarzian and rescaling the entire action with $M$, one then recovers the $2 d$ Lagrangian

$$
L=\pi_{\phi} \phi^{\prime}-\frac{1}{2 M} \pi_{\phi}^{2}+\pi_{f}\left(f^{\prime}-\mathrm{e}^{\phi}\right),
$$


which implies a constant $\pi_{f}=\mu \sim J$ [39, 40], consistent with (21). At finite temperatures the above change of variables results in the additional term $\delta L=-(2 \pi / \beta)^{2} \mathrm{e}^{2 \phi}$ in (22) [41-45]. By contrast, in Refs. [39, 40] a modified finite-temperature relation between $f$ and $\phi$ was used, $(\tan \pi f / \beta)^{\prime}=\mathrm{e}^{\phi}$, which yields the same $1 d$ Liouville Hamiltonian (21) at all temperatures. However, in this case the variable $f$ ceases to be cyclic, which makes the intrinsically $1 d$ nature of this theory more obscure.

Quantizing the 'particle-in-(electro)magneticfield' Hamiltonian (20) for $a=\mathcal{A} / M, b=1 / M$, and $c=\mathcal{A}^{2} / 4 M$, factorizing its eigenstates, $\Psi(\phi, f)=\psi(\phi)$ $\times \mathrm{e}^{\mathrm{i} \mu f}$, and shifting the variable $\phi \rightarrow \phi-\ln \mathcal{A} / \mu$, one arrives at the $1 d$ Schroedinger equation

$$
\left(-\frac{\partial^{2}}{\partial \phi^{2}}+\lambda^{2}\left(\mathrm{e}^{2 \phi} \operatorname{sgn} \mu\right)\right) \psi=\left(\epsilon-\lambda^{2}\right) \psi,
$$

where $\epsilon=2 M(E-c)$ and $\lambda=a / 2 b^{1 / 2}$ (in the finitetemperature case $\lambda=\mu \beta=O(\beta J) \gg 1$ ).

In the previous studies of the SYK model the sign of $\mu=\pi_{f}$ would be routinely chosen negative and the squared exponential term $\mathrm{e}^{2 \phi}$ neglected (alternatively, in Refs. [39, 40] the latter would have never appeared in the first place) so as to reproduce the monotonic repulsive potential of the Liouville Hamiltonian anticipated on the basis of the correspondence with the bulk Euclidean $A d S_{2}$,4-7, 16-24.

For $\mu<0$ the positive definite spectrum of (23) is continuous, $\epsilon_{k}=\left(k^{2}+1 / 4+\lambda^{2}\right)$, parametrized by a 'momentum' $k$, while its eigenstates are given by the Whittaker function (here $z=2 \lambda \mathrm{e}^{\phi}$ )

$$
\psi_{k} \sim \mathrm{e}^{-\phi / 2} W_{\lambda, \mathrm{ik}}(z)
$$

For $b=0$ (24) reduces to the eigenstates of (21) given by the modified Bessel functions, $\psi_{k} \sim K_{2 \mathrm{ik}}(\sqrt{z})$ [39-45].

In terms of the eigenstates $\psi_{k}$ the partition function given by the (non-Gaussian) path integral can be computed as

$$
\begin{aligned}
& Z(\beta)=\int_{\phi(-\beta / 2)=\phi_{0}}^{\phi(\beta / 2)=\phi_{0}} \mathrm{D} \phi \mathrm{e}^{-\int_{\tau} L(\phi)}= \\
& \int_{0}^{\infty} \mathrm{d} k\left|\psi_{k}\left(\phi_{0}\right)\right|^{2} \mathrm{e}^{-E_{k} \beta} .
\end{aligned}
$$

For $b=0$ this calculation yields the free energy of the SYK model [4-7, 16-24, 39, 40]

$$
\begin{aligned}
& F=-\frac{1}{\beta} \ln Z(\beta)= \\
& E_{0}-\frac{S_{0}}{\beta}-\frac{2 \pi^{2} M}{\beta^{2}}+\frac{\pi^{2} \mu N}{6 \beta^{3} J^{2}}+\frac{3}{2 \beta} \ln \beta J,
\end{aligned}
$$

where $E_{0}$ and $S_{0}$ are the extensive ground state energy and zero-temperature ('residual') entropy. The last two terms represent the next order corrections $(O(1 / J \beta)$ and $O(1 / N)$, respectively) [4-7, 16-24.

Using (25) one finds the (many-body) density of states (DOS)

$$
\rho(\epsilon)=\frac{1}{2 \pi \mathrm{i}} \int_{\beta} \mathrm{e}^{\beta E} Z(\beta) \sim \mathrm{e}^{S_{0}} \sinh (2 \pi \sqrt{\epsilon}) .
$$

Alternatively, this result can be inferred from the well-known exact DOS [46, 47], $\rho(\epsilon) \sim \sinh$ $2 \pi \sqrt{\epsilon} /(\cosh 2 \pi \sqrt{\epsilon}+\cos 2 \pi \lambda)$, by shifting the field $\phi \rightarrow \phi-\ln (-2 \lambda)$ and taking the limit $\lambda \rightarrow \mathrm{i} \infty[4-7$, 41-45.

In contrast to the Liouville scenario, for $s g n \mu=1$ Eq. (23) features the non-monotonic Morse potential and may possess additional discrete states given by the associated Laguerre polynomials

$$
\psi_{n}(z) \sim z^{\lambda-n-1 / 2-z / 2} L_{n}^{2 \lambda-2 n-1}(z)
$$

at the discrete energies $\epsilon_{n}=-(n-\lambda+1 / 2)^{2}$, $n=0, \ldots,[\lambda-1 / 2]$.

At low temperatures $(\mu \beta \gg 1)$ the number $[\lambda-1 / 2]$ of the bound states is large and they dominate the partition function. Moreover, their spectrum becomes almost equidistant, allowing one to replace the actual Morse potential with the approximate quadratic ('oscillator') one.

Furthermore, in the presence of the reparametrization symmetry-breaking term (14) the effective action becomes that of a 'damped Morse potential'. Although canonical quantization of a dissipative system can be intrinsically problematic, one can still resort to the path integral approach to study its statistical mechanics and correlations.

In the quadratic approximation, one then obtains the Gaussian action (here $\Omega \sim \mu \beta / M$ )

$$
\delta S=\frac{M}{2} \sum_{n}\left(\omega_{n}^{2}+\Omega^{2}+\Gamma\left|\omega_{n}\right|\right)\left|\phi_{n}\right|^{2} .
$$

Although the quadratic action (29) is gapped, the dynamics of the conformal time $f$ still features 
the zero modes $n=0, \pm 1$ as the deformed Schwarzian action given by Eqs. $(8,14)$ remains invariant under the $S L(2, R)$ group (this can also be inferred from the relation $\left.\phi \approx f^{\prime}-1\right)$.

Using the simplified Eq. (29) one can compute the partition function

$$
\begin{array}{r}
Z(\beta)=\frac{1}{2 \sinh \beta \Omega / 2} \\
\times \prod_{n=1}^{\infty} \frac{\Omega^{2}+\omega_{n}^{2}}{\Omega^{2}+\omega_{n}^{2}+\Gamma\left|\omega_{n}\right|},
\end{array}
$$

where the divergent product can be regularized by introducing the cutoff frequency $\omega_{\max } \sim J$.

This way one obtains the free energy

$$
\begin{aligned}
& \frac{F}{N}=\frac{1}{\beta} \ln (\beta \Omega)+\frac{1}{\beta} \sum_{n=1}^{\omega_{\max }} \ln \left(1+\frac{\Gamma|\omega|}{\omega_{n}^{2}+\Omega^{2}}\right) \approx \\
& \approx \frac{\Omega}{2}+\frac{1}{\beta} \ln \left(1-\mathrm{e}^{-\beta \Omega}\right)+\frac{\Gamma}{2 \pi} \ln \left(\frac{J}{\Omega}\right)
\end{aligned}
$$

for $1 / \beta \ll \Omega, \Gamma$, whereas at higher temperatures the quadratic approximation fails and the leading part of Eq. (26) would be reproduced instead. The thermodynamics properties of the Morse model are, therefore, markedly different from those of the Lioville one. In particular, the specific heat is exponentially suppressed.

From (31) one infers the oscillator-like 'Dirac comb' DOS which averages out to a constant at energies $E \gg \Omega$,

$$
\rho(\epsilon) \approx \frac{\lambda-1 / 2}{2 \pi} \sum_{n} \delta\left(E_{n}-\Omega\left(n+\frac{1}{2}\right)\right) \sim M .
$$

For a more detailed comparison with the standard SYK case one can also evaluate the correlator of the stress tensor $T(\tau)=M\left(f^{\prime \prime \prime}-(2 \pi / \beta)^{2} f^{\prime}\right)$

$$
\begin{aligned}
& \langle T(\tau) T(0)\rangle=M \sum_{n} \frac{\mathrm{e}^{2 \pi \tau / \beta}\left(\omega_{n}^{2}-(2 \pi / \beta)^{2}\right) \omega_{n}^{2}}{\omega_{n}^{2}+\Omega^{2}+\Gamma\left|\omega_{n}\right|} \\
& \sim M \max \left[1 / \beta^{3}, \Omega^{3}\right] \sin \Omega \tau \mathrm{e}^{-\Gamma \tau / 2} .
\end{aligned}
$$

At $\tau=0$ this result agrees with the direct estimation of the energy variance (for $\Gamma<\Omega$ )

$$
\left\langle(\delta E)^{2}\right\rangle=\frac{\partial^{2}}{\partial \beta^{2}} \ln Z(\beta) \sim M \max \left[1 / \beta^{3}, \Omega^{3}\right] .
$$

As a more subtle diagnostic of the boundary dynamics, in the 'Schwarzian' (long-time, low-temperature, $M \lesssim \beta$ ) limit the fluctuations of the Liouville soft mode strongly affect the averaged products

$$
\begin{aligned}
& \left\langle G_{f}\left(\tau_{1}, \tau_{2}\right) \ldots G_{f}\left(\tau_{2 p-1} \tau_{2 p}\right)\right\rangle= \\
& =\int \mathrm{D} \phi \prod_{i=1}^{p} \frac{\mathrm{e}^{\Delta\left(\phi\left(\tau_{2 i-1}\right)+\phi\left(\tau_{2 i}\right)\right)}}{\left(\int_{\tau_{2 i-1}}^{\tau_{2 i}} \mathrm{e}^{\phi}\right)^{2 \Delta}} \mathrm{e}^{-\int_{\tau} L(\phi)} .
\end{aligned}
$$

The denominator in (35) can be promoted to the exponent where it contributes to the overall piece-wise (in the time domain) Liouville potential representing the $2 p$ consecutive quenches [39, 40].

As the result, in the original SYK case with $q=2$ the fluctuation-dressed averages $\left\langle G_{f}^{p}(\tau, 0)\right\rangle$ were found to change their $p$-dependent algebraic decay $\sim 1 / \tau^{p / 2}$ for $\tau \ll M$ to the universal behaviour $\sim 1 / \tau^{3 / 2}$ developing for $\tau \gg M[39$, 40].

By contrast, in the Morse theory for $\Omega \ll 1 / M$ the fluctuation-averaged two-point correlator changes its behaviour from (6) for $\tau \ll M$ to

$$
\begin{aligned}
& \left\langle G_{f}(\tau, 0)\right\rangle \approx \sum_{n} \mathrm{e}^{-E_{n} \tau} N_{1}\left(E_{n}\right) \sim \\
& \left\{\begin{array}{l}
\frac{1}{\tau}, M \ll \tau \ll \frac{1}{\Omega}, \\
\mathrm{e}^{-\Omega \tau}, \tau \gg \frac{1}{\Omega},
\end{array}\right.
\end{aligned}
$$

since the matrix element $N_{1}=\left|\left\langle 0\left|\mathrm{e}^{\Delta \phi}\right| n\right\rangle\right|^{2} \sim$ $\left[L_{n}^{n}\left(-\Delta^{2} / 2\right)\right]^{2} \Delta^{2 n}$ and the DOS (32) are non-singular for $n \leq \Sigma \lambda$ and the sum over $\sim \lambda$ terms can be approximated by the integral. In the opposite limit, $1 / M \lesssim \Omega$, there is no room for algebraic behaviour and (36) decays exponentially.

Likewise, the SYK averages of the higher powers $\left\langle G_{f}^{p}(\tau, 0)\right\rangle$ are sensitive to the behaviour of the $p$-particle DOS and under the above conditions demonstrate the crossovers from the short-time power-law behaviour $\sim 1 / \tau^{p / q}$ to the intermediate universal one, $\sim 1 / \tau$, and, finally, to the exponential decay $\sim \mathrm{e}^{-\Omega \tau}$ at the longest times. In the latter limit, the would-be universal algebraic contribution of the continuous part of the spectrum, $1 / \tau^{6 \Delta}$, is suppressed by the much smaller factor $\sim \mathrm{e}^{-\Omega \lambda \tau}$.

In addition to the Schwarzian fluctuations, the multi-point correlators can also receive subleading $O(1 / N)$ contributions from the massive 
modes which are not governed by the Schwarzian action (8) but can be accounted for by summing the ladder diagrams [4-7, 16-24].

Specifically, in the case of the $p=2$ function $\left.\left\langle\mathrm{G}_{f} \tau_{1}, \tau_{2}\right) \mathrm{G}_{f}\left(\tau_{3}, \tau_{4}\right)\right\rangle$ the massive modes do not significantly contribute to $\tau_{2,3}<\tau_{1,4}$, whereas in the domain $\tau_{1,2}<\tau_{3,4}$ they do, being solely responsible for the irreducible (non-factorizable) contribution to that function [39, 40].

In particular, upon analytically continuing from the domain $\tau_{4}<\tau_{2}<\tau_{3}<\tau_{1}$ to the real times $\tau_{1}=\beta / 4-\mathrm{i} t / 2, \tau_{2}=-\beta / 4-\mathrm{i} t / 2, \tau_{3}=\mathrm{i} t / 2$, $\tau_{4}=-\beta / 2+\mathrm{i} t / 2$, the all-important out-of-timeorder (OTO) correlators demonstrate their initial short-time/high-temperature exponential growth

$$
\frac{\left\langle G_{f}\left(\tau_{1}, \tau_{3}\right) G_{f}\left(\tau_{2}, \tau_{4}\right)\right\rangle}{\left\langle G_{f}(\beta / 2,0)\right\rangle^{2}}=1-O\left(\frac{\beta}{M}\right) \mathrm{e}^{\lambda_{t} t}
$$

revealed by summing the 'causal' ladder series for $\beta \ll t \ll \beta \ln M[4-7,16-24$ ].

In the Morse case the counterpart of (37) exhibits the less-than-maximal Lyapunov exponent

$$
\lambda_{L}=\frac{2 \pi}{\beta}(1-O(\gamma))
$$

which should be contrasted with the result obtained in the original SYK model $\lambda_{L}=2 \pi / \beta(1-O(1 / \beta J))$ 4-7, 16-24.

Also, in the intermediate-time regime (present for $\Omega \ll 1 / M$ ) the Morse OTO function decays with the real time $t$ as

$$
\left\langle G_{f}\left(\tau_{1}, \tau_{2}\right) G_{f}\left(\tau_{3}, \tau_{4}\right)\right\rangle \sim 1 / t^{4}
$$

the dependence of which again differs from the $\sim 1 / t^{6}$ asymptotic found in the $q=2$ SYK model [39, 40].

Returning to the general question of the holographic principle's implementation in the SYKlike models, one finds that some intrinsically $1 d$ details of the Schwarzian/Liouville theories can also be observed in their conjectured bulk duals.

Specifically, in the JT theory the same $1 d$ differential equation (23) emerges, its spectrum now being that of the $S L(2, R)$ Casimir operator formulated in terms of two angular variables, $\theta$ and $\varphi$, related to the space-time coordinates $\left(\tan (\theta-\varphi / 2)=r \mathrm{e}^{-\tau}, \tan (\theta+\varphi / 2)=r \mathrm{e}^{\tau}\right)$. The wave function once again factorizes onto the 'angular' and 'radial' ones, $\mathrm{e}^{\mathrm{i} m \varphi} \chi_{k}(\theta)$, the latter obeying the $1 d$ equation [4-7, 16-24] solved by the functions (24),

$$
\left(-\frac{\partial^{2}}{\partial \theta^{2}}-\frac{k^{2}+1 / 4}{\cos ^{2} \theta}+2 \lambda m \tan \theta\right) \chi=\left(m^{2}-\lambda^{2}\right) \chi \cdot(40
$$

Alternatively, the tangle of (pseudo)holographic relationships between the $\operatorname{SL}(2, R)$-symmetric boundary (Schwarzian/Liouville-like) and bulk (JT-like) theories can be viewed as different forms of embedding (at fixed radial and angular vs temporal and angular coordinates, respectively) into the global $\mathrm{AdS}_{3}$ space $41-45,48-52$.

To further elucidate such generalized relationship one can use the framework of the generalized JT-dilaton theory

$$
S=\int_{\tau, r}(R \Phi+U(\Phi)) \sqrt{g}+\int_{\tau} K \Phi,
$$

where the appropriate dilaton potential $U(\Phi)$ might be able to reflect the various $\operatorname{Diff}\left(S^{1}\right)$-symmetry breaking extensions to the basic Schwarzian action.

To that end, the quadratic term $U_{2}(\Phi) \sim \Phi^{2}$ has already been shown to correspond to the non-local contribution (11) [4-7]. In the future, it would be interesting to establish a link between the higher order terms $\Phi^{n}$ and (non)local and/or (non)geometric deformations of the fundamental Schwarzian action (8).

In the meantime, the natural emergence of the 'particle-in-(electro)magnetic-field' (Liouvillelike) quantum mechanics provides a convenient technical framework for generating novel sequences of (pseudo)holographic $1 d$ systems and their (ostensibly) $2 d$ duals. The latter would then be essentially topological, akin to the theory of incompressible electron droplets in the Quantum Hall Effect [53-55].

Still more examples of such correspondence can be discovered by studying other Virasoro orbits as well as the even more general problem of quantum mechanics on a wider class of group manifolds and their cosets [56-58].

To conclude, the systematic (non-conformal) extensions of the SYK model can be achieved along the lines of single-particle quantum mechanics, thus allowing one to further explore their putative bulk (albeit, dimensionally reducible) duals. Continuing such work may help to elucidate the true status of the holographic studies of the concrete (first of all, condensed matter) systems. 


\section{References}

[1] S. Sachdev and J. Ye, Gapless spin-fluid ground state in a random, quantum Heisenberg magnet, Phys. Rev. Lett. 70, 3339 (1993).

[2] S. Sachdev, Holographic metals and the fractionalized Fermi liquid, Phys. Rev. Lett. 105, 151602 (2010).

[3] S. Sachdev, Bekenstein-Hawking entropy and strange metals, Phys. Rev. X 5, 041025 (2015).

[4] A. Kitaev, KITP seminars (2015), https://online. kitp.ucsb.edu/online/joint $98 / \mathrm{kitaev} /$

[5] A. Kitaev, Notes on $\widetilde{S L}(2, R)$ representations, arXiv: 1711.08169

[6] A. Kitaev and S.J. Suh, The soft mode in the Sachdev-Ye-Kitaev model and its gravity dual, J. High Energy Phys. 2018(05), 183 (2018).

[7] A. Kitaev, Statistical mechanics of a two-dimensional black hole, arXiv:1808.07032

[8] E. Witten, An SYK-like model without disorder, arXiv: 1610.09758

[9] R. Gurau, The complete 1/N expansion of a SYKlike tensor model, arXiv:1611.04032

[10]R. Gurau, The $\imath \epsilon$ prescription in the SYK model, arXiv: 1705.08581

[11]I.R. Klebanov and G. Tarnopolsky, Uncolored random tensors, melon diagrams, and the Sachdev-Ye-Kitaev models, Phys. Rev. D 95, 046004 (2017).

[12]S. Giombi, I.R. Klebanov, and G. Tarnopolsky, Bosonic tensor models at large $N$ and small $\varepsilon$, Phys. Rev. D 96, 106014 (2017).

[13]M. Ammon and J. Erdmenger, Gauge/ Gravity Duality: Foundations and Applications (Cambridge University Press, 2015).

[14]J. Zaanen, Y. Liu, Y.-W. Sun, and K. Schalm, Holographic Duality in Condensed Matter Physics (Cambridge University Press, 2015).

[15]S. Hartnoll, A. Lucas, and S. Sachdev, Holographic Quantum Matter (MIT Press, 2018).

[16]J. Maldacena, S.H. Shenker, and D. Stanford, A bound on chaos, J. High Energy Phys. 2016(08), 106 (2016).

[17]J. Maldacena and D. Stanford, Comments on the Sachdev-Ye-Kitaev model, Phys. Rev. D 94(10), 106002 (2016).
[18]J. Maldacena, D. Stanford, and Z. Yang, Conformal symmetry and its breaking in two dimensional nearly anti-de-Sitter space, Prog. Theor. Exp. Phys. 2016(12), 12C104 (2016).

[19]D. Stanford and E. Witten, Fermionic localization of the Schwarzian theory, J. High Energy Phys. 2017(10), 008 (2017).

[20]J. Polchinski and V. Rosenhaus, The spectrum in the Sachdev-Ye-Kitaev model, J. High Energy Phys. 2016(04), 001 (2016).

[21]D.J. Gross and V. Rosenhaus, The bulk dual of SYK: cubic couplings, J. High Energy Phys. 2017(05), 092 (2017).

[22]D.J. Gross and V. Rosenhaus, All point correlation functions in SYK, J. High Energy Phys. 2017(12), 148 (2017).

[23]G. Sárosi, $A d S_{2}$ holography and the SYK model, arXiv:1711.08482

[24]H.W. Lin, J. Maldacena, and Y. Zhao, Symmetries near the horizon, arXiv: 1904.12820

[25]D.V. Khveshchenko, Taking a critical look at holographic critical matter, Lith. J. Phys. 55, 208 (2015).

[26]D.V. Khveshchenko, Demystifying the holographic mystique, Lith. J. Phys. 56, 125 (2016).

[27]S. Caracciolo, M.A. Cardella, and M. Pastore, Remarks on replica diagonal collective field condensations in SYK, arXiv:1807.10213 [hep-th]

[28]I.Ya. Aref'eva, M.A. Khramtsov, M.D. Tikhanovskaya, and I.V. Volovich, On replica-nondiagonal large $N$ saddles in the SYK model, EPJ Web Conf. 191, 06007 (2018).

[29]H. Wang, D. Bagrets, A.L. Chudnovskiy, and A. Kamenev, On the replica structure of SachdevYe-Kitaev model, arXiv:1812.02666

[30]G. Gur-Ari, R. Mahajan, and A. Vaezi, Does the SYK model have a spin glass phase?, arXiv: 1806.10145

[31]D.V. Khveshchenko, Thickening and sickening the SYK model, SciPost Phys. 5, 012 (2018).

[32]D.V. Khveshchenko, Seeking to develop global SYK-ness, Condens. Matter 3(4), 40 (2018).

[33]Y. Gu, X-L. Qi, and D. Stanford, Local criticality, diffusion and chaos in generalized Sachdev-YeKitaev models, J. High Energy Phys. 2017(05), 125 (2017). 
[34]Y. Gu, A. Lucas, and X-L. Qi, Energy diffusion and the buttery effect in inhomogeneous Sachdev-YeKitaev chains, SciPost Phys. 2, 018 (2017).

[35]Y. Gu, A. Lucas, and X.-L. Qi, Spread of entanglement in a Sachdev-Ye-Kitaev chain, J. High Energy Phys. 2017(09), 120 (2017).

[36]Y. Gu and A. Kitaev, On the relation between the magnitude and exponent of OTOCs, J. High Energy Phys. 2019(02), 75 (2019).

[37]V. Bonzom, L. Lionni, and A. Tanasa, Diagrammatics of a colored SYK model and of an SYK-like tensor model, leading and next-to-leading orders, J. Math. Phys. 58(5), 052301 (2017).

[38]D. Benedetti and R. Gurau, 2PI effective action for the SYK model and tensor field theories, J. High Energy Phys. 2018(05), 156 (2018).

[39]D. Bagrets, A. Altland, and A. Kamenev, SachdevYe-Kitaev model as Liouville quantum mechanics, Nucl. Phys. B 911, 191 (2016).

[40]D. Bagrets, A. Altland, and A. Kamenev, Powerlaw out of time order correlation functions in the SYK model, Nucl. Phys. B 921, 727 (2017).

[41]T.G. Mertens, G.J. Turiaci, and H.L. Verlinde, Solving the Schwarzian via the conformal bootstrap, J. High Energy Phys. 2017(08), 136 (2017).

[42]T.G. Mertens, The Schwarzian theory - origins, J. High Energy Phys. 2018(05), 36 (2018).

[43]Z. Yang, The quantum gravity dynamics of near extremal black holes, arXiv:1809.08647

[44]A. Blommaert, T.G. Mertens, and H. Verschelde, The Schwarzian theory - a Wilson line perspective, J. High Energy Phys. 2018(12), 22 (2018).

[45]A. Blommaert, T.G. Mertens, and H. Verschelde, Fine structure of Jackiw-Teitelboim quantum gravity, arXiv:1812.00918

[46] A. Comtet and P.J. Houston, Effective action on the hyperbolic plane in a constant external field, J. Math. Phys. 26, 185 (1985).
[47]A. Comtet, On the Landau levels on the hyperbolic plane, Ann. Phys. 173, 185 (1987).

[48]A. Jevicki, K. Suzuki, and J. Yoon, Bi-local holography in the SYK model, J. High Energy Phys. 2016(07), 7 (2016).

[49]A. Jevicki and K. Suzuki, Bi-local holography in the SYK model: perturbations, J. High Energy Phys. 2016(11), 46 (2016).

[50]S.R. Das, A. Jevicki, and K. Suzuki, Three dimensional view of the SYK/AdS duality, J. High Energy Phys. 2017(09), 17 (2017).

[51]S.R. Das, A. Ghosh, A. Jevicki, and K. Suzuki, Spacetime in the SYK model, J. High Energy Phys. 2018(07), 184 (2018).

[52]S.R. Das, A. Ghosh, A. Jevicki, and K. Suzuki, Three dimensional view of arbitrary $q$ SYK models, J. High Energy Phys. 2018(02), 162 (2018).

[53]M. Laskin, T. Can, and P. Wiegmann, Collective field theory for quantum Hall states, Phys. Rev. B 92, 235141 (2015).

[54]M. Laskin, Y.H. Chiu, T. Can, and P. Wiegmann, Emergent conformal symmetry and geometric transport properties of quantum Hall states on singular surfaces, Phys. Rev. Lett. 117, 266803 (2016).

[55]S. Klevtsov, X. Ma, G. Marinescu, and P. Wiegmann, Quantum Hall effect and Quillen Metric, Commun. Math. Phys. 349, 819-855 (2017).

[56]G. Mandal, P. Nayak, and S.R. Wadia, Coadjoint orbit action of Virasoro group and two-dimensional quantum gravity dual to SYK/tensor models, J. High Energy Phys. 2017(11), 46 (2017).

[57]A. Gaikwad, L.K. Joshi, G. Mandal, and S.R. Wadia, Holographic dual to charged SYK from 3D Gravity and Chern-Simons, arXiv:1802.07746

[58]T.G. Mertens and G.J. Turiaci, Defects in JackiwTeitelboim Quantum Gravity, arXiv:1904.05228

\title{
APIE (PSEUDO)HOLOGRAFINĘ SYK TIPO MODELIŲ PRIGIMTI
}

\author{
D.V. Khveshchenko
}

